\title{
Can dark energy emerge from quantum effects in a compact extra dimension?
}

\author{
A. Dupays ${ }^{1,4,5}$, B. Lamine $e^{2,3,4,5}$, and A. Blanchard ${ }^{4,5}$ \\ ${ }^{1}$ Université de Toulouse, UPS, Laboratoire Collisions Agrégats Réactivité, IRSAMC, 31062 Toulouse, France \\ 2 Université Pierre et Marie Curie, UPMC, ENS, Laboratoire Kastler Brossel, 75252 Paris Cedex 05, France \\ 3 CNRS, UMR 8552, 75252 Paris, France \\ 4 Université de Toulouse, UPS-OMP, IRAP, 31400 Toulouse, France \\ e-mail: brahim. lamine@irap.omp.eu \\ 5 CNRS, IRAP, 14, avenue Edouard Belin, 31400 Toulouse, France
}

Received 8 January 2013 / Accepted 17 April 2013

\section{ABSTRACT}

\begin{abstract}
The origin of the accelerated expansion of the universe is a major problem in both modern cosmology and theoretical physics. In quantum field theory, simple estimations of the vacuum contribution to the density energy of the Universe are known to lead to catastrophically high values compared to observations. A gravitational Casimir effect from an additional compact dimension of space is known to lead to an effective cosmological constant. Nevertheless, such a contribution by itself is usually not regarded as a plausible source for accelerating the expansion, given the constraints on such scenarios. Here, we propose that the Casimir vacuum contribution of the gravitational field actually provides a low positive value to the density energy of the universe. The key new ingredient is to assume that only modes with shorter wavelengths than the Hubble radius contribute to the vacuum energy. Such a contribution gives a positive energy density, has a naturally Lorentz invariant equation of state in the usual 4D spacetime, and can thus be interpreted as a cosmological constant. Its value agrees with observations for a radius of a fifth extra dimension given by $35 \mu \mathrm{m}$. The implied modification of the gravitational inverse square law is close but below existing limits from experiments testing gravity at short range.
\end{abstract}

Key words. gravitation - dark energy - cosmology: theory

\section{Introduction}

The evidence of the accelerated expansion of the universe has grown since the first result from the Hubble diagram of distant type Ia supernovae (Riess et al. 1998; Perlmutter et al. 1999). The angular power spectrum of the fluctuations in the cosmic microwave background and the large-scale properties of the galaxy distribution are all consistent with the accelerated expansion of a homogenous universe, while no alternative Friedmann-Lemaître model seems to be able to reproduce these three data sets (Frieman et al. 2008; Blanchard 2010). Dark energy, the origin of the cosmic acceleration, is often qualified as one of the deepest mysteries in modern physics, and its origin is hard to explain within the standard framework of high-energy physics (Weinberg 1989). This issue has been a tremendous stimulation for the community, producing a rich ensemble of theoretical approaches, while being the target of unprecedented efforts in astrophysical observational strategy, either in the form of ground projects (LSST Science Collaborations et al. 2009) or ambitious space projects like EUCLID (Laureijs et al. 2011).

A genuine cosmological constant $\Lambda$, as introduced by Einstein in 1917, accounts for the observed cosmic acceleration. However, most scientists agree on the lack of theoretical motivation for introducing such a term into the Einstein equations (Amendola et al. 2012). One reason that is often invoked is that $\Lambda$ introduces a new fundamental energy scale if one introduces the Planck constant $\hbar$ :

$E_{\Lambda}=\left((\hbar c)^{3} \rho_{\Lambda}\right)^{1 / 4} \simeq 2 \mathrm{meV}$, with $\rho_{\Lambda}=\Lambda c^{4} /(8 \pi G)$ representing $73 \%$ of the energy content of the Universe. On this energy scale, no exotic physics are a priori expected. Equivalently, a dimensional length scale can be associated:

$\ell_{\Lambda}=\left(\frac{\hbar c}{\rho_{\Lambda}}\right)^{1 / 4} \simeq 83 \mu \mathrm{m}$.

An experimental effort has been devoted to observing any deviation from the gravitational laws (Adelberger et al. 2009) on this scale and below. No anomaly has been observed just below this length scale (Kapner et al. 2007). Of course, as we are going to see, this does not exclude any deviation in the gravitational laws controled by $\ell_{\Lambda}$, because numerical factors could lower the true length scale to a value below $\ell_{\Lambda}$. Finally, we need to mention that if $\Lambda$ is a true fundamental constant, then it is not possible to define a single natural length scale from $\hbar, c, G$, and $\Lambda$, but instead one can have

$\ell=\Lambda^{-1 / 2} f\left(\frac{\hbar G \Lambda}{c^{3}}\right)$

with $f$ an arbitrary function of the dimensionless constant $\frac{\hbar G \Lambda}{c^{3}} \sim$ $2 \times 10^{-122}$. This low a number is nothing more than a reformulation of the cosmological constant problem. Taking $f(x)=1$ leads to a cosmological scale for $\ell$ (the size of a static Einstein universe), and $f(x)=\sqrt{x}$ leads to the Planck length, while $f(x)=x^{1 / 4}$ gives the previously introduced scale $\ell_{\Lambda}$, qualified as the natural dark-energy length scale. This scale is the geometric mean of the two former scales. 
Historically, a physical explanation for the cosmological constant came from identification of this term with a Lorentz invariant vacuum (Lemaitre 1934), which leads to the possibility of a gravitationally active vacuum due to the contribution of zero-point energy. This attractive idea was discussed as early as in the 1920s by Nernst and Pauli (see Straumann 2002; Peebles \& Ratra 2003; Kragh 2011 for a historical presentation) but it was immediately realized that this possibility is plagued by a large discrepancy in the estimated order of magnitude. To avoid dramatic consequences for cosmology, it is usually assumed that those vacuum energies do not gravitate or give a renormalized value that is exactely zero. This is the first cosmological constant problem. We are therefore left with the second cosmological problem of how to explain the low "incremental" positive value observed today. A first original idea has been historically proposed in Zel'Dovich (1967); Zel'dovich (1968), considering $\rho_{\Lambda}$ as being gravitational interaction energy between virtual pairs of the quantum electrodynamic (QED) vacuum. Unfortunately, this elegant propositon could still not explain the low value of the possible cosmological constant. Nowadays, the actual contribution of vacuum to the present-day density of the universe is still the subject of debate in the scientific community.

Here, we focus on the possibility of identifying the cosmological constant with effects from the quantum vacuum. In Sect. 2, we briefly discuss the vacuum contribution problem. In four-dimensional spacetime, it can be argued that this contribution vanishes for a massless field, while for a massive field the expected contribution is much greater than the observed one for any standard fundamental mass scale unless fine tuning is invoked. In Sect. 3. we examine the case of adding extra spatial compact dimensions. Indeed, pioneer papers in the 80s (Appelquist \& Chodos 1983b,a) computed the quantum corrections in the energy density of the vacuum stemming from the presence of such extra dimensions. It has been shown that those quantum corrections correspond to a Casimir effect of the gravitational field induced by the periodic boundary conditions along the extra compact dimensions (Rohrlich 1984), but direct identification of this quantum correction with the cosmological constant (Milton 2001; Gardner 2002; Elizalde 2006b) encountered some difficulties that we recall briefly later. However, we show that including the Hubble scale as a maximum wavelength allowed for quantum vacuum modes of the gravitational field provides a mechanism to generate a positive cosmological constant with one extra dimension. We furthermore show that such a scenario leads to a modification of the gravitational inverse square law on scales that should be accessible by experiments in the near future.

\section{The zero-point energy contribution to the vacuum}

Considering the example of a massive scalar field, the contribution of zero-point energy to the density can be obtained as the vacuum expectation value of the 00 component of the energy momentum tensor $T^{\mu v}(\hbar=c=1)$

$\rho_{v}=\left\langle 0\left|T^{00}\right| 0\right\rangle=\int \frac{\mathrm{d}^{d} \boldsymbol{k}}{(2 \pi)^{d}} \frac{1}{2} \sqrt{\boldsymbol{k}^{2}+m^{2}}$

with $d$ the number of spatial dimensions and $\boldsymbol{k}$ the wave vector. The vacuum pressure can be computed in a similar way to the density:

$p_{v}=(1 / d) \sum_{i}\left\langle 0\left|T^{i i}\right| 0\right\rangle=\frac{1}{d} \int \frac{\mathrm{d}^{d} \boldsymbol{k}}{(2 \pi)^{d}} \frac{1}{2} \frac{\boldsymbol{k}^{2}}{\sqrt{\boldsymbol{k}^{2}+m^{2}}}$.
These contributions are highly divergent and therefore need some regularization treatment. The most trivial regularization procedure would be to introduce an ultraviolet cutoff $k_{\mathrm{c}}$ in momentum space, above which the theory breaks down. Nevertheless, this procedure introduces two flaws: i) the energy density scales as $k_{\mathrm{c}}^{d+1}$, which leads to a catastrophic value compared to the observed energy density in our universe for any scale $k_{\mathrm{c}}$ related to high-energy physics scales; ii) this cutoff in momentum explicitly violates Lorentz-invariance and leads to a vacuum expectation value of the energy-momentum tensor, which is not proportional to $g_{\mu \nu}$ and therefore cannot be accepted as such for a description of vacuum. The inclusion of non-Lorentz invariant counter terms can restore the symmetry and lead to the correct equation of state (Hollenstein et al. 2012). Another convenient approach is to use a covariant regularization, such as the dimensional regularization in which the number of dimensions $d$ is written as $d=D+\epsilon$, with $D$ an integer and $\epsilon \rightarrow 0$. Introducing a constant $\mu$ (the dimension of which is a mass, or the inverse of a length) so that the energy density and pressure keep the correct dimension, one obtains (see for example Martin 2012)

$p=-\rho=\frac{m^{d+1} \Gamma\left(-\frac{d+1}{2}\right)}{\mu^{\epsilon} 2^{d+2} \pi^{\frac{d+1}{2}}}$

For instance, for $D=3$, discarding the diverging $1 / \epsilon$ term and using the modified minimal subtracting scheme, one finally obtains

$p=-\rho=-\frac{m^{4}}{64 \pi^{2}} \ln \left(\frac{m^{2}}{\mu^{2}}\right)$.

It is now explicit that the Lorentz-invariance is preserved (since $p=-\rho$ ). Moreover, the scaling of the energy density is now like $m^{d+1}$, which is better than $k_{\mathrm{c}}^{d+1}$ in the hard cutoff regularization. Nevertheless, the presence of the regulator $\mu$ does not allow a prediction for $\rho$, while natural values for $\mu$ lead to catastrophically high value compared to the observed value of $\rho_{\Lambda}$. In any event, the important point to be stressed at this level is that for a massless field ( $m=0$ ) the contribution to the vacuum energy density is exactly zero so that this regularization procedure accounts for a degravitation of massless fields, even if it does not give any physical mechanism that would be at its origin (see Ellis et al. 2011; Smolin 2009, for one example of such theories). This result corroborates the simple remark that if we were to build a traceless energy momentum tensor from the metric $g^{\mu \nu}$, the only solution would be to have $\left\langle T^{\mu v}\right\rangle=0$. Said differently, to accomodate the equation of radiation (i.e. massless fields), $p=\rho / d$ with the one of vacuum, $p=-\rho$, one needs $p=\rho=0$.

Though the specific consideration for a massless field does not stand for a general demonstration, the previous consideration corroborates the standard conclusion that some mechanism sets the contribution of vacuum energy to exactly zero in an isotropic spacetime of arbitrary dimensions. The origin of the accelerated expansion of the universe is then logically expected to happen by means of a distinct physical mechanism. The late domination of a scalar field or modifications to the Einstein-Hilbert action are the two options most investigated so far, and have been the subject of intensive research activities ever since there is evidence of an accelerated expansion (Clifton et al. 2012; Tsujikawa 2010).

In the next section we present a physical mechanism to generate a nonzero positive density energy and pressure from zeropoint energies of a massless field (the gravitational field itself). This is achieved by assuming the existence of an additional compact spatial dimension, which will therefore modify Eq. (6). It is 
well known that modification of the boundary conditions of a quantum field leads to nontrivial physical properties of the vacuum. The Casimir force between two infinite conducting plates is a famous example of a physical nonzero but finite contribution from the QED vacuum even if the electromagnetic field is massless. In the latter configuration, the isotropy of space has obviously been broken by the presence of boundary conditions. The pressure in the direction normal to the plates satisfies $p_{\perp}=3 \rho$ (with $\rho<0$ ), while the pressure parallel to the plates satisfies $p_{\|}=-\rho$ (Brown \& Maclay 1969), in accordance with the traceless nature of the electromagnetic field. Remarkably enough, the Lorentz invariance in the two dimensions parallel to the plates ensures the equation of state $p_{\|}=-\rho$ with a nonzero value of $\rho$. As we will see, in the presence of additional compact dimensions of space, a gravitational Casimir effect allows for a nonzero density energy that is Lorentz invariant in the usual 4D spacetime $(p=-\rho)$, even for a massless (traceless) field.

\section{Casimir effect from a higher compact dimension}

The existence of additional space dimensions has been considered with various purposes in modern physics (for a review, see for example Rubakov 2001), from the Kaluza-Klein scenario (Kaluza 1983) aiming at unifying interactions to the more recent braneworld paradigm dealing with the hierarchy issue (Arkani-Hamed et al. 1998; Antoniadis et al. 1998; Randall \& Sundrum 1999). In this picture, matter is localized in a 4D spacetime (the brane), while gravity can propagate in all the dimensions (the bulk). This picture allows for a large extra dimension (see also Antoniadis 1990, for a first proposal using large extra dimensions). Because the gravitational field is massless, dimensional regularization (Eq. (6)) ensures that the energy density vanishes in arbitrary $N$-dimensional infinite isotropic spacetime. However, in the case of compact additional dimensions, the situation is different since the structure of the quantum vacuum is modified by the quantification of the gravitational field in the additional dimensions. This quantification of the gravitational-field modes in the bulk leads to a Casimir energy that was computed many years ago for one extra dimension in pioneer works from the 80s in Appelquist \& Chodos (1983b, a) for a Minkowski background metric and later in Rohrlich (1984) using a zero-point energy calculation and an exponential cutoff regularization.

In what follows, we first reproduce the calculation for $N=1$ using dimensional regularization. We thus assume the existence of one spatial additional dimension compactified on a circle of radii $R$. The periodic condition $f\left(x^{i}, x^{4}+2 \pi R\right)=f\left(x^{i}, x^{4}\right)$ allows the metric tensor to be expanded in Fourier series:

$g_{\mu \nu}\left(x^{i}, x^{4}\right)=\sum_{n=-\infty}^{\infty} g_{\mu \nu}^{(n)}\left(x^{i}\right) \exp \left(\operatorname{in} x^{4} / R\right)$

where $x^{4}$ is the coordinate in the extra dimension. In a specific gauge, the metric satisfies the propagation equation $\nabla^{2} g_{\mu \nu}=0$ so that the gravitational modes $g_{\mu \nu}^{(n)}$ satisfy the dispersion relation:

$\omega_{n}(\boldsymbol{k})=\sqrt{\boldsymbol{k}^{2}+n^{2} / R^{2}}$.

The mode $n=0$ is the usual massless graviton, while the excited modes $n \neq 0$ correspond to effective massive gravitational fields of masses $n / R$ (Kaluza-Klein tower). To simplify, we model the gravitational field by a scalar field and multiply the final result by the number of polarization states $p_{m}=m(m-3) / 2$ in $m$-dimensional spacetime $\left(p_{5}=5\right)$. The previous assumption is justified since we consider a flat extra dimension. For situations with curvature, a conformally coupled scalar field would have been a better description of the true gravitational field.

With a vacuum energy per mode given by $\omega_{n} / 2$, the total vacuum energy density is obtained as

$\rho=\frac{p_{d+2}}{2 \pi R} \sum_{n=-\infty}^{\infty} \int \frac{\mathrm{d}^{d} \boldsymbol{k}}{(2 \pi)^{3}} \frac{1}{2} \sqrt{\boldsymbol{k}^{2}+n^{2} / R^{2}}$.

Each term in the previous sum can be dimensionally regularized using Eq. (6) :

$\rho=-\frac{2 p_{d+2} \Gamma\left(-\frac{d+1}{2}\right)}{(4 \pi)^{\frac{d+3}{2}} R^{d+2}} \zeta_{\mathrm{R}}(-d-1)$

where $\zeta_{R}$ is the Zeta Riemann function. Using the reflection formula

$\Gamma\left(\frac{z}{2}\right) \zeta_{\mathrm{R}}(z) \pi^{-z / 2}=\Gamma\left(\frac{1-z}{2}\right) \zeta_{\mathrm{R}}(1-z) \pi^{(z-1) / 2}$,

one finally obtains a finite (regularized) contribution for $d=3$

$\rho_{\text {App }}=-\frac{15 \zeta_{\mathrm{R}}(5)}{128 \pi^{7} R^{5}} \simeq-4 \times 10^{-5} \frac{1}{R^{5}}$.

This expression agrees with previous studies based on different regularization schemes: hard cutoff in Appelquist \& Chodos (1983b), exponential cutoff in Rohrlich (1984), or point-splitting in Milton (2001). The density in the brane is then obtained by a trivial integration (in the fifth dimension):

$\rho_{\text {brane }}=-\frac{15 \zeta_{\mathrm{R}}(5)}{64 \pi^{6} R^{4}} \simeq-2.5 \times 10^{-4} \frac{1}{R^{4}}$.

Generalizations to a spacetime structure $M^{4} \times S^{N}$ have been done in Candelas \& Weinberg (1984) and Chodos \& Myers (1985) for odd $N$ and later in Myers (1986) and Kantowski \& Milton (1987) for even $N$. Such generalizations to extra dimension $N$ can be written as

$\rho_{\text {brane }}=\kappa_{N} \frac{\hbar c}{R^{4}}$

and are summarized in Table 1. The second colum gives the relation between the gravitational Casimir energy $\rho$ and the radius of the extra dimension in $R^{4} \times S^{N}$ compactification for different values of $N$ (the differences with Candelas \& Weinberg (1984) and Chodos \& Myers (1985) being the polarization factors $p_{d+2}$ ). The third column gives the radius of the extradimension, such that this gravitational Casimir energy is equal to the observed dark energy density (the sign being the one of the normalization constant in Col. 2). The fourth column gives the size of the additional dimension that would solve the hierarchy problem (i.e. in order to have a Planck scale equal to $1 \mathrm{TeV}$ ). Finally, the last column summarizes the present observational constraints on the size of this extra dimension, from $N=1$ to $N=7$ extra dimensions (Beringer et al. 2012; Fermi-LAT Collaboration et al. 2012). For instance, one can see from this table that the hierarchy problem can only be solved with $N \geq 6$. The conclusion to be drawn from this table is that extra dimensions cannot solve the hierarchy problem and explain the origin of dark energy at the same time without any additional ingredient. We now assume that the extra dimension is not introduced to solve the hierarchy 
problem. Then, if one identifies the cosmological constant with the Casimir energy, it is clear that odd values of $N$ are still excluded. Even values of $N$ greater than one are more problematic, since the evaluation of their contribution contains a logarithmic term of some unknown scale $\mu$. This makes the normalization constant (Col. 2) not well determined. Nevertheless, any plausible value of $\mu$ (say below the Planck mass) will not make a large numerical difference and will therefore lead to a radius that is not very different from those obtained in the odd case. Therefore we are left with the only possibility of having one extra dimension if the cosmological constant has to be created only from the Casimir energy contribution of a higher compact dimension. However, in the 1D case, as can be seen from Table 1, a negative sign then seems to be obtained for $\rho$, while observations request a positive sign. More sophisticated and somewhat speculative scenarios have been proposed to overcome this dead end, although no completely convincing solution has emerged (Milton 2003; Elizalde 2006a). Among those scenarios, we mention the possibility of considering a nontrivial topology of space (Elizalde 2006b,a) or different boundary conditions (twisted boundary condition will change the sign of the Casimir energy Milton 2003 , as well as $\mathbb{Z}^{2}$ symmetry on a torus compactification $T^{2}$ with certain shape moduli $\theta$, as shown in Matsuda $\&$ Seki 2006). They also comprise new fermion fields in the bulk (Greene \& Levin 2007; Matsumoto 2013), massive graviton (Bauer \& Seidl 2005), a latticed extradimension (Cognola et al. 2004, 2005), a time evolution of the size of the extra dimension (Leon 2005; Blanchard et al. 2012) or even of the scale factor (Cahill 2011; Bernard \& LeClair 2013), curvature in the bulk (for example anti-de Sitter and a massive scalar field as in $\mathrm{Li}$ 2005, or a bulk fermion field in the Randall-Sundrum model, Shao \& Chen 2010). In conclusion, previous attemps to directly identify this Casimir energy with the cosmological constant have not led to a definite conclusion.

In the following we re-examine this question in the cosmological context. We show that this provides a mechanism leading to a positive Casimir energy density $\rho$ at late times. The key ingredient is to take the finite age of the Universe into account. This finite age implies the existence of a length scale, the Hubble radius $c H(t)^{-1}$. This clearly adds a boundary condition that has to be considered. We make two assumptions to account for this effect. First, only modes corresponding to wavelengths shorter than the Hubble radius $c H(t)^{-1} \sim c t$ contributes to the density of the vacuum energy (see also Cahill 2011 for a similar proposition in the cosmological context, or Padmanabhan 2012 for a different mechanism also implying the Hubble radius). The second assumption is that as long as the Hubble radius is shorter than the radius $R$ of the extra dimension, the energy density is equal to zero. The reason is that when the horizon is smaller than the radius of the extra dimension, the structure of the quantum vacuum cannot depend on the compact nature of the extra dimension because gravitons have not yet explored the "compactness" of space. The situation should therefore be equivalent to the one previously discussed of a massless scalar field in an isotropic spacetime, leading to $\left\langle T_{\mu \nu}\right\rangle=0$ (see Eq. (6)). It is easy to see why those assumptions can yield a net positive contribution of zeropoint energy. Indeed, when the horizon radius crosses the radius of the extra dimension, the change in the vacuum is only due to new modes that appear with a wavelength larger than $2 \pi R$. Those modes contribute with $\hbar \omega / 2$ of vacuum energy and a UV cutoff of about $1 / R$, leading to a finite positive contribution. In this picture, the cosmological constant can be seen as a "temporal" Casimir effect, as if the boundary conditions were switched on at a given moment of time. The observable quantity is therefore the change of vacuum energy when the Hubble radius crosses the extra dimension.

The previous discussion implies that (13) has to be changed in order to fix the subtration point in the energy density at $t=$ $R / c$. To perform this task, we add a low-energy cutoff $\omega_{n}(\boldsymbol{k})>$ $2 \pi / t$ to (10) and a counterterm $C T(t)$, which restores Lorentz invariance and insures that $\rho$ is zero as long as $t \leq R / c$,

$$
\rho(t)=\frac{5}{R} \int_{\omega_{n}(\boldsymbol{k})>2 \pi / t} \frac{\mathrm{d}^{3} \boldsymbol{k}}{(2 \pi)^{3}} \sum_{n=-\infty}^{\infty} \frac{1}{2} \sqrt{\boldsymbol{k}^{2}+n^{2} / R^{2}}+C T(t)
$$

with $C T(t)$ such that $\rho(t \leq 2 \pi R / c)=0$. At later times, the boundary condition changes and the energy density is no longer kept to zero. The counterterm then stays equal to its value at time $2 \pi R / c$ (which we note $C T$ ), obtained from the transition condition $\rho(2 \pi R / c)=0$

$$
\frac{5}{R} \int_{\omega_{n}(k)>0}(\ldots)-\frac{5}{R} \int_{\omega_{n}(k)<c / R}(\ldots)+C T=0 .
$$

In the late time regime, $t \gg R / c$, the cut off introduced by the Hubble radius can be neglected so that the present-day energy density $\rho_{0}$ reads as

$$
\begin{aligned}
\rho_{0} & =\frac{5}{R} \int_{\omega_{n}(\boldsymbol{k})>0} \frac{\mathrm{d}^{3} \boldsymbol{k}}{(2 \pi)^{3}} \sum_{n=-\infty}^{\infty} \frac{1}{2} \sqrt{\boldsymbol{k}^{2}+n^{2} / R^{2}}+C T \\
& =\frac{5}{R} \int_{\omega_{n}(\boldsymbol{k})<c / R} \frac{\mathrm{d}^{3} \boldsymbol{k}}{(2 \pi)^{3}} \sum_{n=-\infty}^{\infty} \frac{1}{2} \sqrt{\boldsymbol{k}^{2}+n^{2} / R^{2}}
\end{aligned}
$$

after using Eq. (17). We see in the previous expression that $R$ acts as a UV cutoff for the sum of zero-point energies. The condition $\omega_{n}(k)^{2}<(1 / R)^{2}$ implies that only the term $n=0$ contributes to the last integral, rendering it elementary. This allows us to obtain the value of the density (reintroducing explicitely $\hbar$ and $c$ ):

$\rho_{0}=\frac{5 \hbar c}{32 \pi^{3} R^{5}}$.

The other components of the energy-momentum tensor can be obtained from $\rho_{0}$. Indeed, the traceless nature of the gravitational field, together with the symmetry of the problem, requires that

$\left\langle T^{\mu v}\right\rangle=\rho_{\text {cas }}\left(g^{\mu v}+5 \hat{n}^{\mu} \hat{n}^{v}\right)$

with $\hat{n}^{\mu}$ the unit spacelike vector pointing in the extra dimension $\left(\hat{n}^{2}=-1\right)$ and $\rho_{\text {cas }}$ is a constant (because of the conservation laws $\left.\partial_{\mu} T^{\mu v}=0\right)$. One finds that the pressure in the extra dimension (perpendicular to the brane) is $p^{\perp}=4 \rho_{0}$, while the pressure in the brane (the usual spacetime dimension) is such that $p^{\|}=-\rho_{0}$. This situation is analogous to the previous discussion of the electromagnetic Casimir situation (Sect. 2). Also $p^{\perp}$ could have been derived from energy conservation when considering a variation in the radius $R$. On the brane, the energy-momentum tensor is obtained by integrating over the fifth dimension,

$\rho_{\text {brane }}=\frac{5 \hbar c}{16 \pi^{2} R^{4}}, \quad p_{\text {brane }}=-\rho_{\text {brane }}$.

Equation (21) can thus be identified with the present-day dark energy density $\rho_{\mathrm{DE}}=0.7 \rho_{\mathrm{c}} \approx 4 \mathrm{keV} / \mathrm{cm}^{3}$ for an appropriate value of $R$. Such an identification leads to predicting the size of the extra dimension given by

$R=\left(\frac{5 \hbar G}{2 \pi c^{3} \Lambda}\right)^{\frac{1}{4}}=35 \mu \mathrm{m}$ 
Table 1. Summary of constraints on extra dimensions.

\begin{tabular}{ccccc}
\hline \hline$N$ & $\kappa_{N}=\frac{R^{4} \rho}{\hbar c}$ & $\begin{array}{c}R_{N}^{\Lambda} \\
(\mu \mathrm{m})\end{array}$ & $\begin{array}{c}R_{N}^{\text {hierar. }} \\
(\mu \mathrm{m})\end{array}$ & $\begin{array}{c}\text { Constraints } \\
(\mu \mathrm{m})\end{array}$ \\
\hline 1 & $-2.5 \times 10^{-4}$ & $(10.5)$ & $2.6 \times 10^{19}$ & $\begin{array}{c}<44 \text { (ISL) } \\
<44 \text { (NS) } \\
<30 \text { (ISL) } \\
2\end{array}$ \\
& - & - & $2.2 \times 10^{3}$ & $<0.00016$ (NS) \\
3 & $1.1 \times 10^{-3}$ & 15.0 & $9.7 \times 10^{-3}$ & $<2.6 \times 10^{-6}(\mathrm{NS})$ \\
4 & - & - & $2.0 \times 10^{-5}$ & $<10^{-3}(\mathrm{LHC})$ \\
5 & $1.2 \times 10^{-2}$ & 27.2 & $5.0 \times 10^{-7}$ & $<1.0 \times 10^{-7}(\mathrm{NS})$ \\
6 & - & - & $4.3 \times 10^{-8}$ & $<4.4 \times 10^{-8}(\mathrm{NS})$ \\
7 & $3.6 \times 10^{-2}$ & 36.1 & $7.3 \times 10^{-9}$ & $<2.4 \times 10^{-8}(\mathrm{NS})$ \\
\hline
\end{tabular}

Notes. ISL is for inverse square law test (Adelberger et al. 2009), NS for neutron star contraints (Hannestad \& Raffelt 2003), and LHC from the CMS experiment at CERN.

A consequence of the present discussion is that gravitational laws are modified on the scale of the radius $R$, which is precisely the range of present experiments, such as the inverse square law tests (Kapner et al. 2007; Adelberger et al. 2009) or experiments aiming at measuring the Casimir force (Antoniadis et al. 2011). More interestingly, because of numerical prefactors, the value of $R$ predicted here is slightly lower than the dimensional length scale $\ell_{\Lambda}$ introduced in the introduction.

The 4D gravitational potential, in the presence of one extra dimension is obtained as an infinite sum of Yukawa potentials, each of them corresponding the one massive mode of the Kaluza Klein tower (Arkani-Hamed et al. 1999; Kehagias \& Sfetsos 2000)

$V=-\frac{G_{3} M}{r} \sum_{m=-\infty}^{\infty} \mathrm{e}^{-|m| \frac{r}{R}}=-\frac{G_{3} M}{r} \operatorname{coth}\left(\frac{r}{2 R}\right)$.

For $r \gg R$, the previous expression is given by the Newtonian expression $(m=0)$ plus the contribution of the lightest KaluzaKlein modes $(n= \pm 1)$,

$V \simeq-\frac{G_{3} M}{r}(1+2 \exp (-r / R)), \quad r \gg R$.

This corresponds to a Yukawa modification with strength $\alpha=2$ and a range given by the radius $R$ of the extra dimension. Using this type of potential, the analyses of ISL tests (Adelberger et al. 2009) give a maximum size of $44 \mu \mathrm{m}$ for $R$ at $95 \%$ confidence level. Our prediction is therefore just below the present-day limits. Improvement of these measurements will therefore be critical for testing our model; nevertheless, when probing the ISL at distance $\sim R$, the complete expression should be used instead of the simple Yukawa description (see Fig. 1). On smaller scales, the best constraints on gravity laws are obtained by Casimir force measurements (Decca et al. 2007). The experiments are performed at a smaller distance than the size of the extra dimension, leading to a different behavior for the potential (23)

$V \simeq-\frac{2 G_{3} M R}{r^{2}}$

It leads to a power-law modification of the gravitational force between two test masses with an amplitude scaled by $R$ given by Eq. (22). This modification could be searched for in Casimir experiments operating at short distances, although present-day limits in those experiments are still several orders of magnitude above our prediction (Antoniadis et al. 2011).

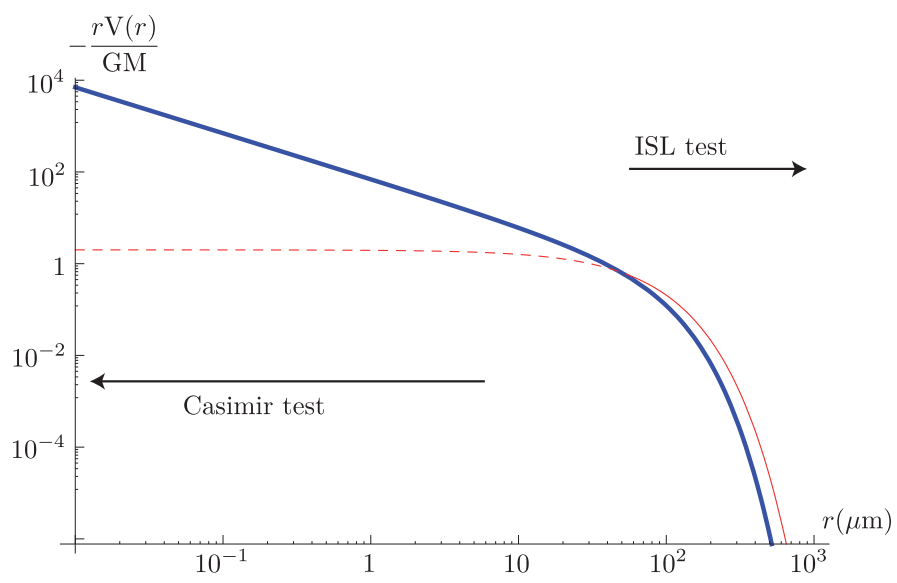

Fig. 1. Point-particle gravitational potential for one extra dimension (bold black line) and Yukawa (red line). The Yukawa modification is taken with a range given by the present-day constraint stemming from Adelberger et al. (2009), $\lambda=44 \mu \mathrm{m}$. The dashed part of this curve corresponds to scales not tested in Adelberger et al. (2009). The extradimension potential is plotted for a value of the radius given by $R=35 \mu \mathrm{m}$. Our prediction is not excluded by experiments, but further improvement will soon give a definite answer. The short-scale behavior is different from the pure Yukawa modification usually searched for in experiments.

\section{Conclusion}

The zero-point energy from a quantized field present in an additional compact dimension naturally provides a nonvanishing value for the vacuum contribution to density of the universe, through a Casimir-like effect. Such a term is naturally Lorentz invariant in the usual 4D spacetime and therefore may provide a natural explanation for the observed cosmological constant. However, present-day experimental limits on possible additional dimension, summarized in Table 1, exclude more than one extra dimension for such contribution to be the only origin of the observed dark energy density ${ }^{1}$. The case of one extra dimension is still allowed, although in the case of a Minkowski spacetime, it leads to a negative contribution to the density of the universe.

1 Strictly speaking this conclusion holds only for odd number of extra dimensions, as the actual Casimir contribution for even number of extra dimensions is not properly known. Of course, if other contributions are present (brane tensions, warping etc...), large extra dimensions are still viable. 
In the cosmological context, we have proposed that the Hubble radius acts as a cut-off to mode wavelenths contributing to the vacuum expectation value, i.e. an infrared cut-off in Eq. (4). Using a zero-point energy calculation, we showed that this infrared cut-off yields a positive contribution. Therefore this mechanism naturally explains the origin of the observed cosmic acceleration that appears as a manifestation of the quantized gravitational field in an additional dimension. A first consequence of this model is that the Planck energy scale is lowered to $\sim 10^{9} \mathrm{GeV}$. A second consequence is that the equation of state of cosmological dark energy should be exactly that of a cosmological constant, i.e. $w=-1$. A third consequence is that gravitation law would be modified on scales that are about the size of the compact dimension, which is $35 \mu \mathrm{m}$, a value below the purely dimensional dark energy length scale $\left(\hbar c / \rho_{v}\right)^{1 / 4} \sim$ $85 \mu \mathrm{m}$ (Kapner et al. 2007; Beane 1997) and below but close to present experimental limits on departure to the inverse square law of gravity at short scales. This leaves open the fascinating possibility that tests of the gravitation law on short distance shed new light on the nature and origin of cosmic acceleration.

Acknowledgements. The authors are grateful to Serge Reynaud, Carlo Rizzo, and Bertrand Chauvineau for fruitful discussions.

\section{References}

Adelberger, E. G., Gundlach, J. H., Heckel, B. R., Hoedl, S., \& Schlamminger, S. 2009, Progr. Part. Nucl. Phys., 62, 102

Amendola, L., Appleby, S., Bacon, D., et al. 2012 [arXiv: 1206. 1225]

Antoniadis, I. 1990, Phys. Lett. B, 246, 377

Antoniadis, I., Arkani-Hamed, N., Dimopoulos, S., \& Dvali, G. 1998, Phys. Lett. B, 436, 257

Antoniadis, I., Baessler, S., Büchner, M., et al. 2011, Comptes Rendus Physique, 12,755

Appelquist, T., \& Chodos, A. 1983a, Phys. Rev. D, 28, 772

Appelquist, T., \& Chodos, A. 1983b, Phys. Rev. Lett., 50, 141

Arkani-Hamed, N., Dimopoulos, S., \& Dvali, G. 1998, Phys. Lett. B, 429, 263

Arkani-Hamed, N., Dimopoulos, S., \& Dvali, G. 1999, Phys. Rev. D, 59, 086004

Bauer, F., \& Seidl, G. 2005, Phys. Lett. B, 624, 250

Beane, S. R. 1997, General Relativity and Gravitation, 29, 945

Beringer, J., Arguin, J.-F., Barnett, R. M., et al. 2012, Phys. Rev. D, 86, 010001

Bernard, D., \& LeClair, A. 2013, Phys. Rev. D, 87, 063010

Blanchard, A. 2010, The astronomy and astrophycis review, 18, 595

Blanchard, A., Dupays, A., \& Lamine, B. 2012, in Proc. XLVIIth Rencontres de Moriond, 158

Brown, L. S., \& Maclay, G. J. 1969, Phys. Rev., 184, 1272

Cahill, K. 2011 [arXiv: 1102 . 5572]

Candelas, P., \& Weinberg, S. 1984, Nucl. Phys. B, 237, 397
Chodos, A., \& Myers, E. 1985, Phys. Rev. D, 31, 3064

Clifton, T., Ferreira, P. G., Padilla, A., \& Skordis, C. 2012, Phys. Rep., 513, 1

Cognola, G., Zerbini, S., Elizalde, E., Nojiri, S., \& Odintsov, S. 2004, Mod. Phys. Lett. A, 19, 1435

Cognola, G., Elizalde, E., \& Zerbini, S. 2005, Phys. Lett. B, 624, 70

Decca, R. S., López, D., Fischbach, E., et al. 2007, Eur. Phys. J. C, 51, 963

Elizalde, E. 2006a, in The Dark Side of the Universe, eds. C. Manoz, \& G. Yepes, AIP Conf. Ser., 878, 232

Elizalde, E. 2006b, J. Phys. A Math. General, 39, 6299

Ellis, G. F. R., van Elst, H., Murugan, J., \& Uzan, J.-P. 2011, Classic. Quant. Grav., 28, 225007

Fermi-LAT Collaboration, Ajello, M., Baldini, L., et al. 2012, J. Cosmol. Astropart. Phys., 2, 12

Frieman, J. A., Turner, M. S., \& Huterer, D. 2008, ARA\&A, 46, 385

Gardner, C. L. 2002, Phys. Lett. B, 524, 21

Greene, B., \& Levin, J. 2007, J. High Energ. Phys., 2007, 096

Hannestad, S., \& Raffelt, G. G. 2003, Phys. Rev. D, 67, 125008

Hollenstein, L., Jaccard, M., Maggiore, M., \& Mitsou, E. 2012, Phys. Rev. D, 85,124031

Kaluza, T. 1983, in Unified Field Theories of $>4$ Dimensions, eds. V. De Sabbata, \& E. Schmutzer, 427

Kantowski, R., \& Milton, K. A. 1987, Phys. Rev. D, 36, 3712

Kapner, D. J., Cook, T. S., Adelberger, E. G., et al. 2007, Phys. Rev. Lett., 98, 021101

Kehagias, A., \& Sfetsos, K. 2000, Phys. Lett. B, 472, 39

Kragh, H. 2011 [arXiv: 1111.4623]

Laureijs, R., Amiaux, J., Arduini, S., et al. 2011 [arXiv: 1110. 3193]

Lemaître, G. 1934, Proc. Nat. Acad. Sci., 20, 12

Leon, J. P. 2005, General Relativity and Gravitation, 37, 53

Li, L.-X. 2005, Mod. Phys. Lett. A, 20, 733

LSST Science Collaborations, Abell, P. A., Allison, J., et al. 2009 [arXiv:0912.0201]

Martin, J. 2012, Comptes Rendus Physique, 13, 566

Matsuda, S., \& Seki, S. 2006, Int. J. Mod. Phys. A, 21, 3095

Matsumoto, J. 2013 [arXiv: 1303 . 4067]

Milton, K. 2001, The Casimir Effect: Physical Manifestations of Zero-Point Energy (World Scientific)

Milton, K. A. 2003, Gravit. Cosmol., 9, 66

Myers, E. 1986, Phys. Rev. D, 33, 1563

Padmanabhan, T. 2012 [arXiv: 1210.4174]

Peebles, P. J., \& Ratra, B. 2003, Rev. Mod. Phys., 75, 559

Perlmutter, S., Aldering, G., Goldhaber, G., et al. 1999, ApJ, 517, 565

Randall, L., \& Sundrum, R. 1999, Phys. Rev. Lett., 83, 3370

Riess, A. G., Filippenko, A. V., Challis, P., et al. 1998, AJ, 116, 1009

Rohrlich, D. 1984, Phys. Rev. D, 29, 330

Rubakov, V. A. 2001, Physics Uspekhi, 44, 871

Shao, S.-H., \& Chen, P. 2010, Phys. Rev. D, 82, 126012

Smolin, L. 2009, Phys. Rev. D, 80, 084003

Straumann, N. 2002 [arXiv: gr-qc/0208027]

Tsujikawa, S. 2010, in Lect. Notes Phys. (Berlin: Springer Verlag), ed. G. Wolschin, 800, 99

Weinberg, S. 1989, Rev. Mod. Phys., 61, 1

Zel'Dovich, Y. B. 1967, Sov. J. Exp. Theoret. Phys. Lett., 6, 316

Zel'dovich, Y. B. 1968, Sov. Phys. Uspekhi, 11, 381 